\title{
An approach to identify the functional transduction and transmission of an activated pathway
}

\author{
WANG NingQian ${ }^{\dagger}$, BO Lin ${ }^{\dagger}$, ZHANG Fan, TAN XiaoDong, YANG XiaoLi \& XIAO ZhongJu* \\ Department of Physiology, School of Basic Medicine, Southern Medical University, Guangzhou 510515, China
}

Received November 15, 2011; accepted March 13, 2012; published online January 18, 2013

\begin{abstract}
In this study, we investigated the features of latency-amplitude $(L-A)$ functions at different sound frequencies, using extracellular recording from auditory neurons in the central nucleus of the inferior colliculus (ICC) in mice. Isofrequency $L-A$ functions from single neurons could be fit with a newly developed equation based on Pieron's law. The high degree of fitness indicates that the curvatures of all isofrequency $L-A$ functions for a given neuron are similar, and that the difference between $L-A$ functions is due to a shift in their positions in the coordinate system. When we normalized the $L-A$ functions to match the position of the $L-A$ function obtained at the neuronal characteristic frequency (CF), all isofrequency $L-A$ functions from a given ICC neuron were highly superimposed. The similar shapes of the $L-A$ functions at different frequencies may reflect the physical laws of sound being transferred into bioelectric signals. The position of a non-CF $L-A$ function could be measured as the differences of the asymptotic $L$ and $A$ ( $\Delta L$ and $\triangle A$ ) compared to the $L-A$ function at a reference frequency such as the CF. The nerve fibers and synapses connecting to a neuron for acoustic information processing can be functionally simplified as a single "wire" (as the total length of nerve fibers) and "joint" (as the summated size/strength of synapses). The wire and joint mediate information transmission and transduction, respectively. Thus, $\Delta L$ and $\Delta A$ may be measurements of the total length of nerve fibers and the strength of summated synapses in the activated auditory pathway. $\Delta L$ and $\Delta A$ differed between frequency channels and neurons, suggesting that the differences of acoustic neuronal responses are always caused by activation of different pathways, and that the pathways that process sounds are diverse.
\end{abstract}

central nucleus of inferior colliculus, acoustic response, transmission efficacy, transduction efficacy

Citation: Wang N Q, Bo L, Zhang F, et al. An approach to identify the functional transduction and transmission of an activated pathway. Chin Sci Bull, 2013, 58: 1274-1284, doi: $10.1007 / \mathrm{s} 11434-012-5452-0$

Sound perception is affected by four basic components: the travel of sound through the air, the frequency-specific response of the basilar membrane, the propagation of action potentials along axons, and signal processing at synapses [1]. Once the sound arrives at the ear, peripheral and central auditory systems are involved in auditory processing. Both serial and parallel processing of acoustic information occur in the central auditory nervous system [2-5]. This involves a large number of nerve fibers and synapses, connecting to an auditory neuron directly or indirectly (Figure 1(a1)). Theoretically, acoustic responses of all these basic components, including each activated nerve fiber and synapse,

$\dagger$ These authors contributed equally to this work.

*Corresponding author (email: xiaozj@ fimmu.com) should be recorded to study the activated pathway. Although the quantity of nerve fibers and synapses onto a neuron activated by a given acoustic stimulus (AS) are certain, it is impossible to measure anatomically the lengths of the nerve fibers and the strengths of the synapses, because of recording technique limitations.

Although the acoustic pathway comprises several different physical media (air, liquid (e.g., perilymph and endolymph), axons, pre- and post-synaptic membranes, and the synaptic cleft), only two functional processes are involved: (1) the travel of signals through one medium, and (2) the transfer of signals between media. These processes can be considered as "wires" and "joints". Each type of physical medium is considered a wire, whereas each process that 
(a1)

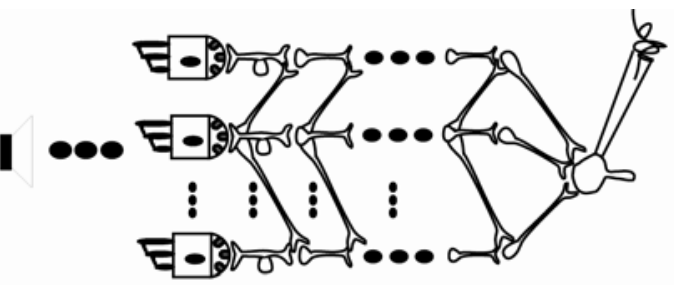

(a2)

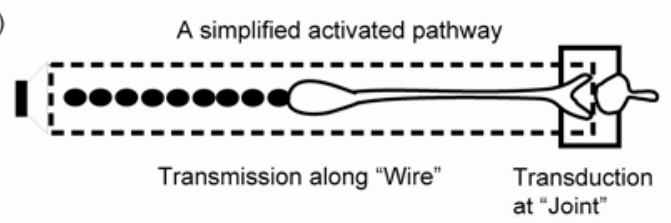

(b)

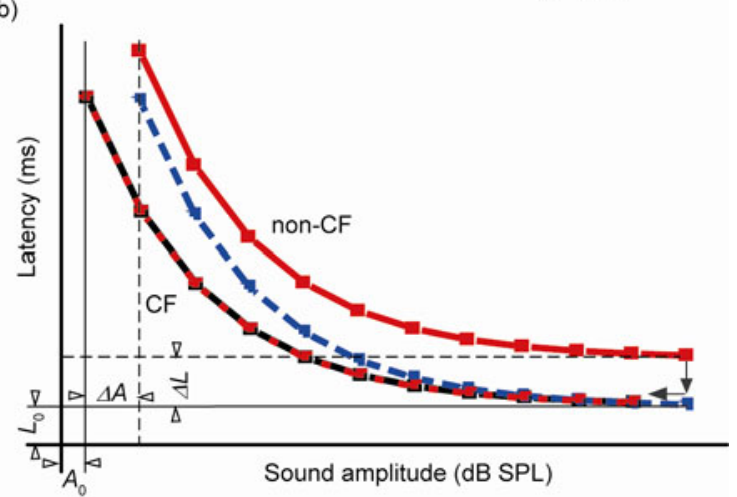

Figure 1 Schematic illustration of the pathway from the sound source to a central auditory neuron (a) and our hypothesis about the superimposition of isofrequency $L-A$ functions (b). (a1) Schematic representation of the nerve fibers and synapses connecting to a neuron that responds to a given AS. (a2) Simplified representation of the activated pathway as a single wire through which transmission occurs (dashed rectangle) and a joint where transduction occurs (rectangle). (b) Our hypothesis about superimposition of $L-A$ functions. The $L-A$ curve for a non-characteristic frequency (CF) AS (red) can be superimposed on the CF $L-A$ curve (black) if it is moved over $\Delta L$ along the $y$ axis (blue) then over $\Delta A$ along the $x$ axis. $L_{0}$ and $A_{0}$ indicate the horizontal and vertical asymptotes, respectively.

occurs between two different media, such as pre-synaptic neurotransmitter release or the post-synaptic membrane response, is treated as a joint. In this study, we defined the signal traveling along the wire as "transmission", and the transfer of the signal at the joint as "transduction". To separate the effects of transmission and transduction, we simplified the structures involved in the acoustic pathway functionally into one wire and one joint (Figure 1(a2)). The wire and joint may be regarded as the total length of the fibers and the total strength of synapse, respectively. Despite this, no methods exist to analyze the functional transmission and transduction of an activated pathway.

Neuronal spike timing, generally measured as the latency of the first acoustic evoked spike $(L)$, has recently received increasing attention as a potential neural code for sensory representation [1,6,7]. Evidence for this has been found in several systems, including somatosensory [8], olfactory [9], auditory [10,11], visual [12], and in the hippocampus [13]. $L$ is more precise and reliable than average spike rates or counts alone in the representation of sound stimulus information
[11,14-17], although this might not be the case for all stimuli and neurons [18]. Therefore, $L$ might provide information on the functional quantity of activated nerve fibers and synapses for a given neuron responding to a certain AS.

Generally, neurons in the central auditory nervous system respond at the shortest delay when the AS is at or near their characteristic frequency $(\mathrm{CF}$; the $\mathrm{AS}$ frequency to which a neuron is most sensitive. That is, the lowest sound amplitude is needed to evoke the neuronal response). And $L$ is positively related to the difference between the AS frequency and the CF of the neuron $[11,16,19,20]$. $L$ decreases as the amplitude $(A)$ of the AS increases, and can be plotted as a function of the AS amplitude ( $L-A$ function) [15-17,21,22]. The $L-A$ functions for acoustic stimuli of different frequencies, such as $\mathrm{CF}$ and non-CF stimuli, are similar in shape but shifted in location on the coordinate system (Figure 1(b)) $[12,17]$. The $L-A$ function can be well described by Pieron's law [23], as well as by other models derived from Pieron's law [11,17,24-28].

The basic form of Pieron's law [23] is:

$$
L-L_{0}=b P^{-a} \text {, }
$$

where $L_{0}$ is the minimum response latency and $P$ is sound pressure in Pascal. $L_{0}, a$, and $b$ are fit constants.

In these models, the first-spike latency is separated into two components (eq. (1)): the stimulus-dependent latency $\left(b P^{-a}\right.$ ) and the constant stimulus-independent minimum latency $\left(L_{0}\right)$ which is the horizontal asymptote of the $L-A$ function [11] (Figure 1(b)). $L_{0}$ was referred to as the "transmission delay" in our previous study [17], and reflects the transmission efficacy from the sound source to the recorded neuron. It comprises sound travel time in the air, frequency-specific delays in the response of the basilar membrane, the fixed delay of synapses, and axonal action potential propagation time. In contrast, the stimulus-dependent latency $\left(b P^{-a}\right)$ is the $L$ corrected for the theoretical minimum $L\left(L-L_{0}\right)$, and is a function of the AS sound pressure.

Considering the $L-A$ curve, there seems to be another constant that describes the response properties along the $x$ axis. We carefully analyzed our published data on the neuronal response to an AS at the CF [17], and found that the response can be fit by a modified model derived from Pieron's law with four fit constants (Appendix I):

$$
L-L_{0}=\lambda e^{-\left(A-A_{0}\right) / \tau},
$$

where $A$ is the stimulus sound pressure and $A_{0}$ is the minimum sound pressure level (dB SPL). $L_{0}, A_{0}, \tau$, and $\lambda$ are fit constants. At the CF, $L_{0}$ is the horizontal asymptote, and $A_{0}$ is the vertical asymptote (Figure 1(b)).

In this modified model, the stimulus-dependent latency $\left(\lambda e^{-\left(A-A_{0}\right) / \tau}\right)$ is a value relative to a constant that is dependent on $A_{0}\left(\lambda e^{A_{0} / \tau}\right)$. The vertical asymptote $A_{0}$ represents the sensitivity threshold of the pathway to the AS, and therefore reflects the reliability of transduction between 
different media [17]. If the pathway is functionally simplified into one wire and one joint (Figure 1(a2)), $A_{0}$ can be seen as a parameter that describes the transduction efficacy, i.e., the summed efficacy of pre-synaptic neurotransmitter release and the post-synaptic membrane responses for all activated synapses. $A_{0}$ and $L_{0}$ determine the position of the $L$ - $A$ curve, while $\tau$ and $\lambda$ determine the curvature.

When neurons responded to two different AS frequencies (e.g., $\mathrm{CF}$ and nearby non-CF) varying in amplitude, two isofrequency $L-A$ functions were obtained (Figure 1(b), [16]). We hypothesized that these two curves could be superimposed after normalization by moving one of them horizontally and vertically (Figure 1(b)). If this is the case, a neuron will respond in a similar way to different AS frequencies, with unchanged values of $\tau$ and $\lambda$ (i.e., similar curvatures), and with the differences originating solely from changes in $A_{0}$ and $L_{0}$. The differences of $L_{0}$ and $A_{0}$ between the two $L-A$ functions may be calculated by fitting the data with eq. (2) using the same values of $\tau$ and $\lambda$ or with eq. (3) with the same values of $L_{0}, A_{0}, \tau$ and $\lambda$.

$$
L-\left(L_{0}+\Delta L\right)=\lambda e^{-\left[A-\left(A_{0}+\Delta A\right)\right] / \tau},
$$

where $L_{0}, A_{0}, \lambda$, and $\tau$ are the constants obtained when the CF $L-A$ data were fit with eq. (2). $\Delta A$ and $\Delta L$ are the constants from this fit, and indicate the differences between the asymptotes $L_{0}$ and $A_{0}$ of the two functions (Figure 1(b), the dashed lines).

According to the quality and meaning of $L_{0}$ and $A_{0}, \Delta L$ and $\triangle A$ may be interpreted as the differences in transmission and transduction efficacy of the activated nerve fibers and synapses in the two frequency-specific pathways converging onto the neuron (Figure 1(a)).

The inferior colliculus is an important nucleus with no analogs in other sensory neuronal systems. It receives convergent inputs from the parallel ascending streams of auditory information [29]. We have previously studied neuronal processing of sound in the central nucleus of inferior colliculus (ICC) using $L$ as the measurement of the acoustic response $[16,17,20,30]$. The features of the ICC neuronal acoustic response include relatively low levels of spontaneous firing, an onset firing pattern, and reproducibility of the first spike latency across different frequencies and amplitudes of the AS. Therefore, we used the $L-A$ curve obtained from the response of ICC neurons fit by our newly developed eqs. (2) and (3) to test our "superimposition of $L-A$ functions" hypothesis. We tried to develop a method to study the relative efficacy of transmission and transduction, the functional differences between frequency channels, and the properties of frequency channels of ICC neurons.

\section{Materials and methods}

\subsection{General}

Forty-five healthy BALB/c mice of either sex (12-18 g; 4-6 weeks old) without hearing defects were provided by the
Experimental Animal Center of the Southern Medical University, Guangzhou, China. The methods for animal preparation, acoustic stimulation, data acquisition, and processing were similar to our previous study [20]. The study was approved by the Animal Care and Use Committee of the Southern Medical University.

\subsection{Surgical preparation}

Atropine sulfate $(0.25 \mathrm{mg} / \mathrm{kg}$, s.c., Sigma-Aldrich, St Louis, MO, USA) was administered at the start of the experiment to reduce bronchial mucous secretion. Anesthesia was induced with sodium pentobarbital (60-70 mg/kg, i.p., Sigma) and maintained by supplemental doses $(13 \mathrm{mg} / \mathrm{kg})$ of the same anesthetic being administered every hour or on indication by the pedal withdrawal reflex.

The mouse was held in a polyethylene foam body-mold box placed in a double-walled soundproof room (temperature maintained at $24-26^{\circ} \mathrm{C}$ ). The head was fixed to a stereotaxic apparatus with a $1.5-\mathrm{cm}$ nail attached to the dorsal surface of the skull vertically with dental cement. A $2 \mathrm{~mm} \times$ $2 \mathrm{~mm}$ area was opened on the cranium, and the dura was removed under a surgical microscope (WPI, Sarasota, FL, USA) to expose the IC of one hemisphere for extracellular recording. Vaseline was used to cover the exposed brain and the pinnae were maintained as in normal awake animals during recordings.

\subsection{Acoustic stimulation}

A Tucker-Davis Technologies System 3 (TDT 3, Tucker-Davis Technologies, Alachua, FL, USA) was used to generate pure tone bursts as the AS. They were applied to the animal by a free-field loudspeaker (ES1, frequency range $2-110 \mathrm{kHz}$ ) located $30 \mathrm{~cm}$ from the front of the head. The loudspeaker was calibrated using $1 / 8$ and $1 / 4$ inch microphones (Brüel and Kjaer 4138, 4135, Naerum, Denmark) and an amplifier (Brüel and Kjaer 2610) at the start of the experiment. The duration of the acoustic pure tone bursts was $50 \mathrm{~ms}$ and the rise-fall time was $5 \mathrm{~ms}$. Pure tone bursts were played using a computer running BrainWare software, and the frequency and amplitude were controlled manually or automatically.

\subsection{Data acquisition}

Glass micropipettes were filled with $2 \mathrm{~mol} / \mathrm{L}$ sodium acetate and $3 \%$ pontamine sky blue $(\sim 1 \mu \mathrm{m}$ tip diameter; impedance: 10-20 M $\Omega$ ), and used for extracellular recording of ICC neuronal activity. The signal was amplified $(\times 2000-10000)$, band-pass filtered $(0.3-3 \mathrm{kHz})$, and processed using the TDT 3 .

The shapes and feature spaces (1st peak to 2 nd peak) of action potentials were stored and monitored during data acquisition to isolate single units by their similar action potential shapes. 
Frequency-amplitude scans were performed using frequencies of $\mathrm{CF} \pm 5 \mathrm{kHz}$ varied in 0.5 or $1 \mathrm{kHz}$ steps, and amplitudes of 10-90 dB above the minimum threshold (MT) varied in 5-20 dB steps. The MT was defined as the AS amplitude that elicited a spike with a probability of 0.1 at the CF [31]. Each AS was applied randomly 15 times at $1 / \mathrm{s}$. After recording, pontamine sky blue was applied to the recording site by microiontophoresis $(-20 \mu \mathrm{A}, 15 \mathrm{~min}$; Neurophore BH-2, Holliston, MA, USA) to confirm the recording site. Data from neurons outside the ICC were discarded.

\subsection{Data processing}

The frequency-amplitude scan provided a frequencyamplitude field of an ICC neuron, from which the basic features of the neurons, CF and MT, were identified.

In a frequency-amplitude field of a neuron, the points containing one or more spikes in response to the 15 presentations of a given AS were used to generate a post-stimulus time histogram, in which an appropriate analysis window including only onset responses was selected to calculate the mean $L( \pm \mathrm{SD})$ and spike count (SC) [17]. The $L-A$ and SCA curves for each AS frequency were plotted, as well as the $L$-frequency and SC-frequency curves for each AS amplitude.

Since the $\mathrm{CF}$ is an important parameter describing the features of a recorded auditory neuron, we used the re-
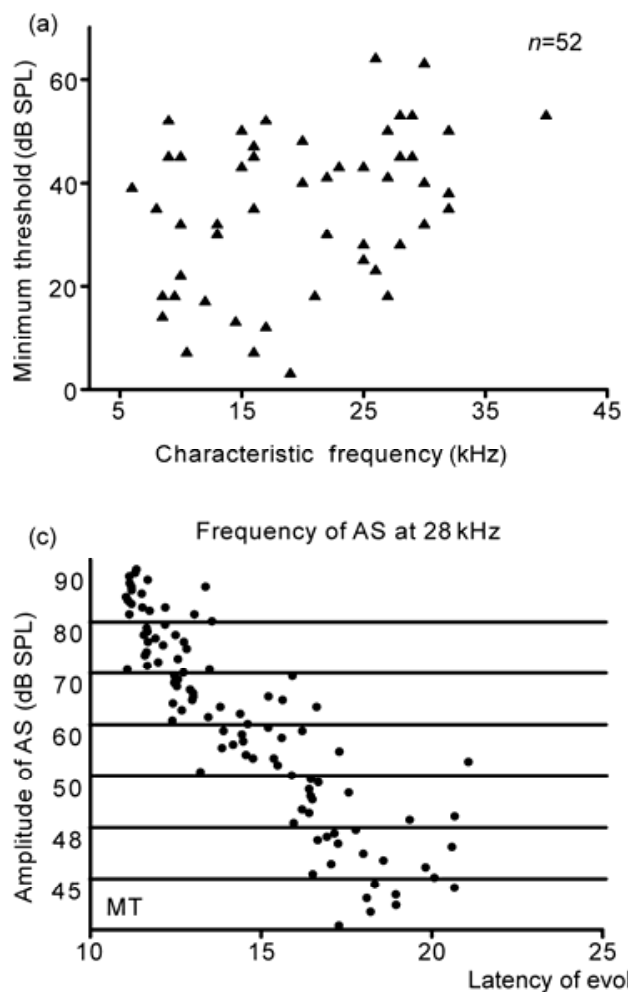

Figure 2 Basic features of ICC neuronal responses to pure tone bursts. (a) There was no correlation between the CF and the MT. (b) The AS frequencies used to test the neuronal response were normally distributed around the CFs (Gaussian Fit, $R^{2}=0.996$ ). (c) Raster plot of response latencies of an ICC neuron at its CF $(28 \mathrm{kHz})$ at different amplitudes of the AS (MT: $45 \mathrm{~dB}$ SPL). (d) Raster plot of the responses of the same neuron at different AS frequency at $70 \mathrm{~dB}$ SPL. sponses to the AS at the $\mathrm{CF}$ as the reference for processing

First, the $L-A$ curve for $C F$ acoustic stimuli was fit using eq. (2) to obtain the constants $L_{0}, A_{0}, \lambda$, and $\tau$. Then, the $L-A$ curves for the non-CF acoustic stimuli in the same neuron were fit with eq. (3) using the four constants $L_{0}, A_{0}, \lambda$, and $\tau$ from the first fit, to obtain $\Delta A$ and $\Delta L$. Finally, we plotted $\Delta A$ and $\Delta L$ against the AS frequency normalized to the $\mathrm{CF}$ (AS frequency - CF).

Microsoft Excel 2003 and OriginPro 7.5 were used for calculating the values of the relevant parameters and for data fitting and plotting. One-way ANOVA in SPSS 11 (SPSS Inc., Chicago, IL, USA) was used to test the difference.

\section{Results}

\subsection{General}

Extracellular recordings were obtained at recording depths of 320-1794 $\mu \mathrm{m}$ from 52 ICC neurons with single-peak frequency-amplitude responses. The CFs of these neurons ranged from 6 to $40 \mathrm{kHz}(20 \pm 8.3 \mathrm{kHz})$ and their MTs were between 3 and $64 \mathrm{~dB}$ SPL $(35 \pm 14.9 \mathrm{~dB}$ SPL). Most recorded ICC neurons ( $n=51 / 52$ neurons) fired at the onset, and only one neuron fired over the entire duration of the AS. There was no correlation between the CF and MT of the recorded ICC neurons (Figure 2(a)). Acoustic responses

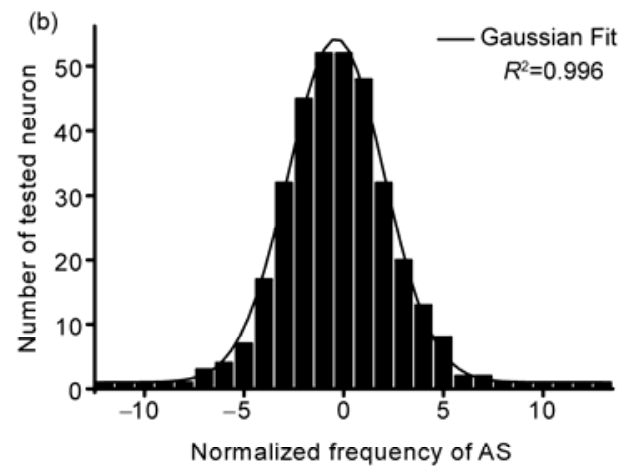

(d) Amplitude of AS at $70 \mathrm{~dB}$ SPL

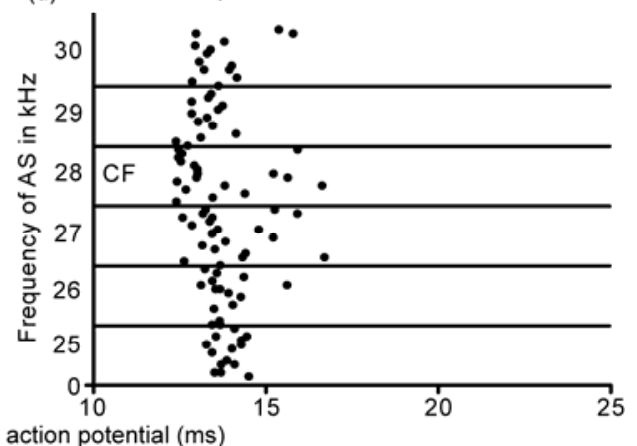
the datasets in this study. 
were tested at frequencies that were normally distributed around the CF of the ICC neurons (Gaussian Fit, $R^{2}=0.996$; Figure 2(b)). The responses occurred at a shorter $L$ for $C F$ stimuli compared to non-CF stimuli (Figure 2(d)), and $L$ increased when the AS amplitude decreased (Figure 2(c)).

\subsection{First spike latency versus spike count}

The $L$ and SC were plotted as isoamplitude SC-frequency and $L$-frequency diagrams (Figure $3(\mathrm{a}),(\mathrm{b})$ ) and as isofrequency SC-amplitude and $L$-amplitude plots (Figure 3(c) and (d)). The SCs of the neuronal responses showed large variance and did not well reflect the amplitude and frequency of the AS (Figure 3(a) and (c)). However, both the isoamplitude $L$-frequency (Figure $3(\mathrm{~b})$ ) and the isofrequency $L-A$ plots (Figure $3(\mathrm{~d})$ ) showed accurate, stable, and reliable relationships between stimuli and responses.

According to criteria based on the monotonicity ratio (SC at the highest amplitude/SC at the best amplitude of the AS $[32,33]$ ), neuronal SC-A responses to the pure tone bursts in the present study could be divided into monotonic (monotonicity ratio $\geqslant 0.8 ; n=24 / 52$ neurons; $46.2 \%$ ) and nonmonotonic (monotonicity ratio $<0.8 ; 28 / 52$ neurons; $53.8 \%$ ). The isoamplitude SC-frequency and $L$-frequency functions used the same raw data as the isofrequency SC-A and $L-A$ functions. Thus, we could only compare the isofrequency $\mathrm{SC}-\mathrm{A} / L-A$ functions of the monotonic amplitude-response neurons to the non-monotonic ones. In both cases, the $L-A$ functions showed more accurate, stable, and reliable results (Figure 4(b) and (d)) than SC-A functions
(Figure 4(a) and (c)). The $L-A$ function showed a similar appearance regardless of whether the response was nonmonotonic or monotonic.

These results were consistent with previous reports $[16,34,35]$. In all isofrequency $L-A$ functions, $L$ decreased with increasing AS amplitudes. The position of the $L-A$ functions differed for different AS frequencies (Figures 3(d), 4(b) and (d)), but the functions were similar in shape.

\subsection{Superimposition of $L-A$ functions}

The CF and non-CF $L-A$ curves $(n=346)$ for all recorded neurons were fit well with eqs. (2) and (3), respectively $\left(R^{2}>0.90\right.$ for 46 recorded ICC neurons). The fits yielded the two constants $\Delta A$ and $\Delta L$, which describe the differences between the asymptotes $L_{0}$ and $A_{0}$ for the non-CF and $\mathrm{CF}$ $L-A$ curves. Using the obtained values of $\Delta A$ and $\Delta L$, all the non-CF $L$ - $A$ curves were normalized by subtraction $(L-\Delta L$ and $A-\triangle A$ ). Then, the CF and non-CF $L-A$ curves of each neuron were re-plotted together, and the normalized non-CF data were fit to eq. (2). The normalization process amounted to moving the non-CF $L-A$ curve horizontally and vertically over a distance of $\Delta A$ and $\Delta L$, preserving the AS-dependent component of $L$, i.e., the shape of the curve. Figure 5(a)-(c) illustrates that the non-CF and CF $L-A$ curves were highly superimposed after this procedure $\left(R^{2}\right.$ values: $0.99,1.00$ and 0.99).

The data from most of the recorded ICC neurons $(n=$ 50/52 neurons) showed good fits, with $R^{2}>0.85$ (mean $R^{2}=$ $0.95 \pm 0.07$; Figure 6$)$. The $L-A$ curves for a relative small
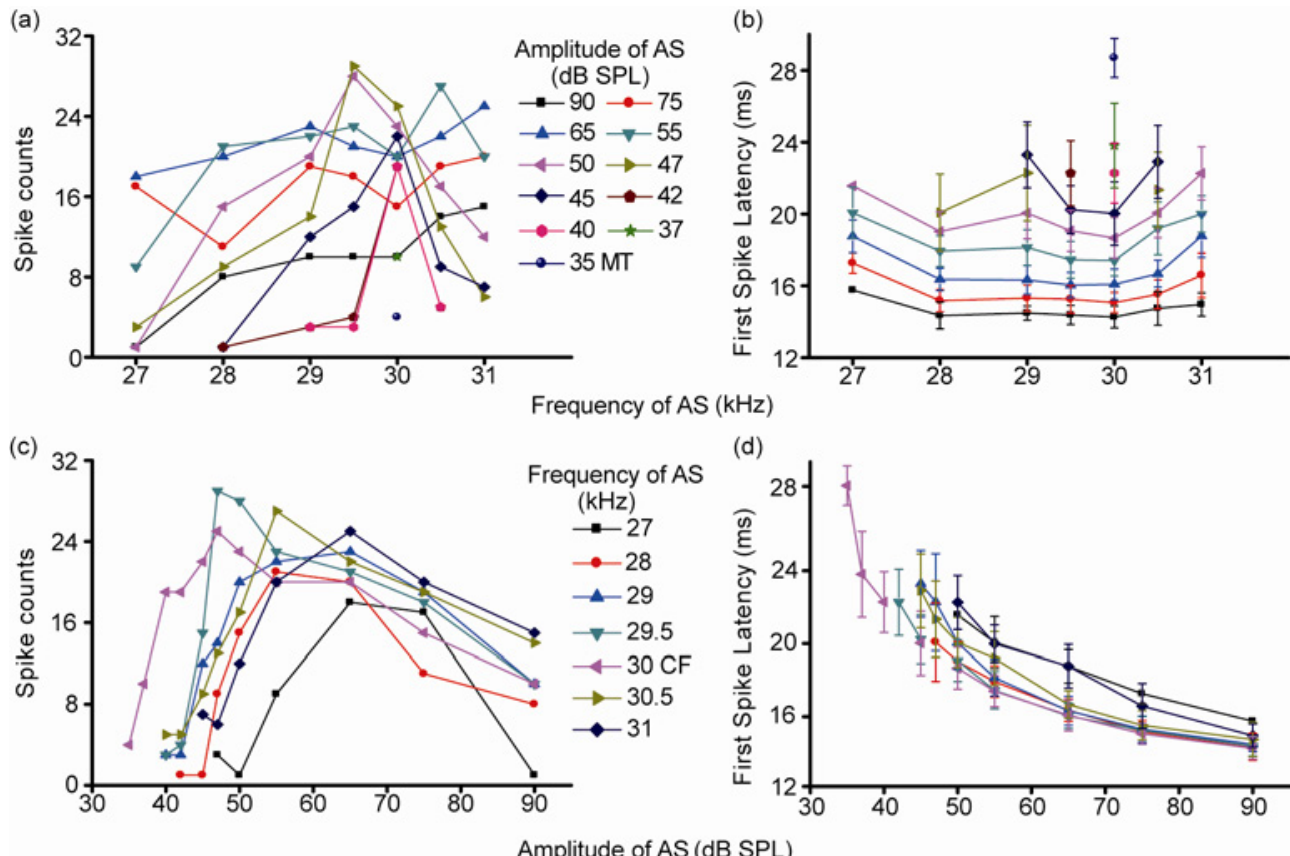

Figure 3 Comparison of the SC and $L$ responses of an ICC neuron (No. 032401; CF: $30 \mathrm{kHz}$. MT: $35 \mathrm{~dB}$ SPL) to pure tones varying in frequency and amplitude. (a) and (b) The isoamplitude SC-frequency (a) and $L$-frequency (b) functions. (c) and (d) The isofrequency SC-A (c) and $L$ - $A$ (d) functions. 

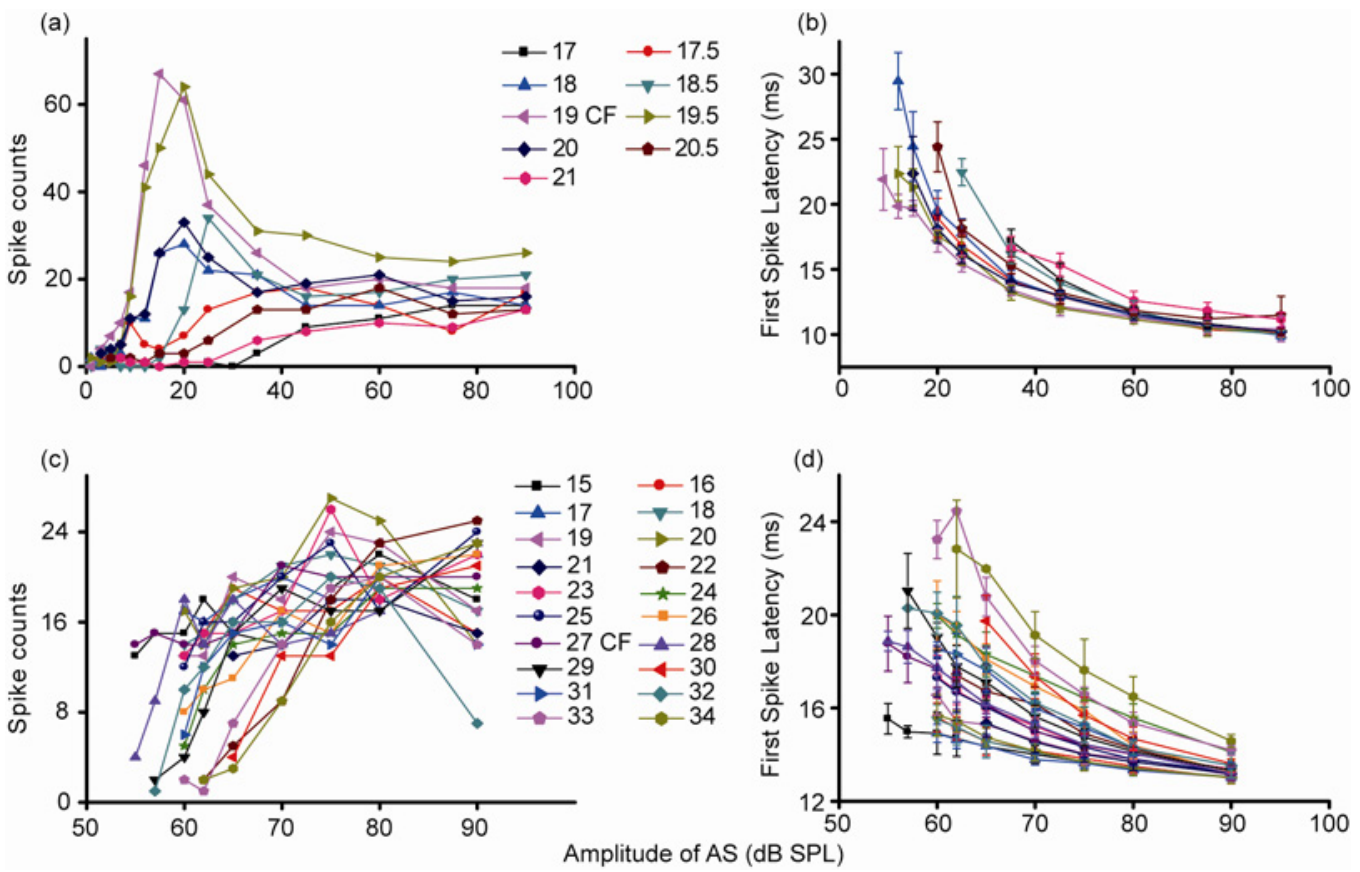

Figure 4 Comparison of isofrequency SC-A ((a) and (c)) and L-A ((b) and (d)) plots in one non-monotonic neuron (No. 070702; CF: 19 kHz; MT: 3 dB SPL) ((a) and (b)) versus one monotonic neuron (No. 033001; CF: 27 kHz; MT: $50 \mathrm{~dB}$ SPL) ((c) and (d)).
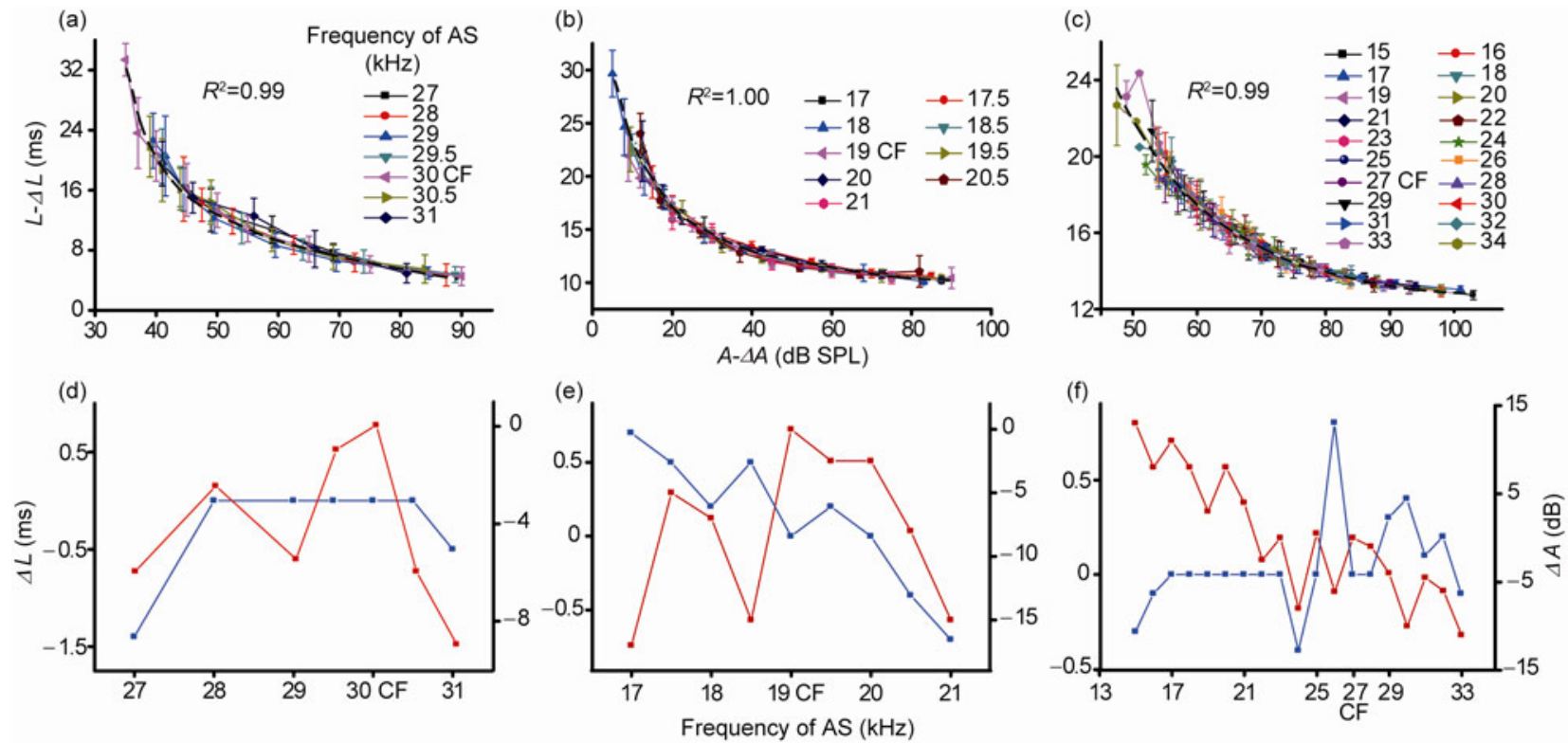

Figure 5 Three examples of superimposition of $L-A$ functions (data from the same neurons as in Figures 3 and 4 ). Superimposed $L-A$ functions for neurons No. 032401 (a), 070702 (b), and 033001 (c) are shown. (d)-(f) Plots show $\Delta A$ (red line, secondary $y$ axis) and $\Delta L$ (blue line, primary $y$ axis) obtained from data fitting using eq. (3) as a function of AS frequency.

range of frequencies including $\mathrm{CF}$ and non-CF had similar shapes while only their positions in the coordinate system were different. This indicates that an ICC neuron responds to pure tone stimuli of different frequencies and amplitudes in an identical way.

According to our hypothesis, $\Delta L$ and $\Delta A$ can be interpreted as the difference in transmission and transduction efficacy of two different frequency channels. Therefore, we plotted the values of $\Delta A$ (red lines) and $\Delta L$ (blue lines) obtained from a given neuron against the stimulus frequency (Figure 5(d)-(f)), to check for any regular pattern in the processing of frequency and amplitude. However, the $\triangle A$ (Figure 5(d)-(f); red lines) and $\Delta L$ (Figure 5(d)-(f); blue lines) were different at different frequencies. This may mean that stimuli at different frequencies activate different sets of synapses and fibers. 


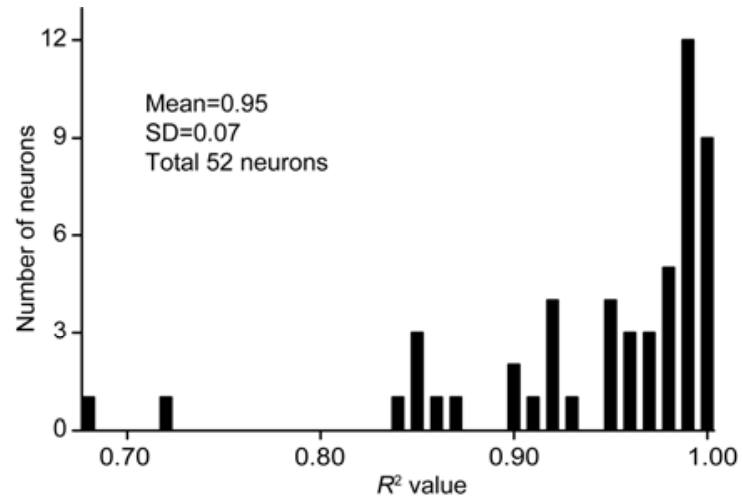

Figure 6 Histogram of the distribution of $R^{2}$ values for the 52 recorded ICC neurons.
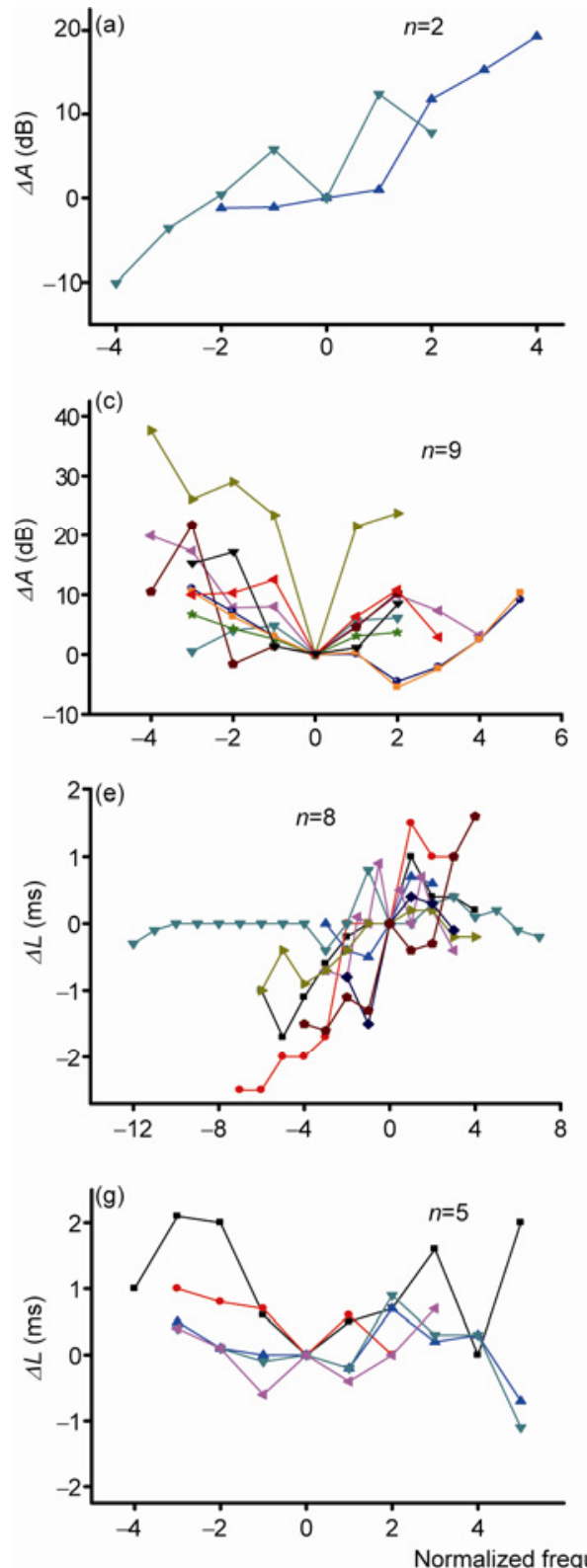

\subsection{Properties of ICC neurons expressed by $\Delta A$ and $\Delta L$}

The fibers and synapses connecting to a neuron activated by a given AS frequency can be treated as a frequency channel. To find some regularity of frequency channels in the ICC neuron population, we plotted $\Delta A$ and $\Delta L$ against the AS frequencies normalized to the $\mathrm{CFs}$ for all recorded neurons, and categorized the changes in $\Delta A$ and $\Delta L$ (Figure 7). ICC neurons that had not been tested at more than two non-CF frequencies were excluded $(n=15 / 52)$. In the remaining 37 neurons, we identified four categories of $\Delta A$ (Figure 7 , upper panels) or $\Delta L$ (Figure 7 , lower panels) when plotted against AS frequency. The $\Delta A$ or $\Delta L$ increased monotonically with increasing frequency for type I (Figure 7(a) and
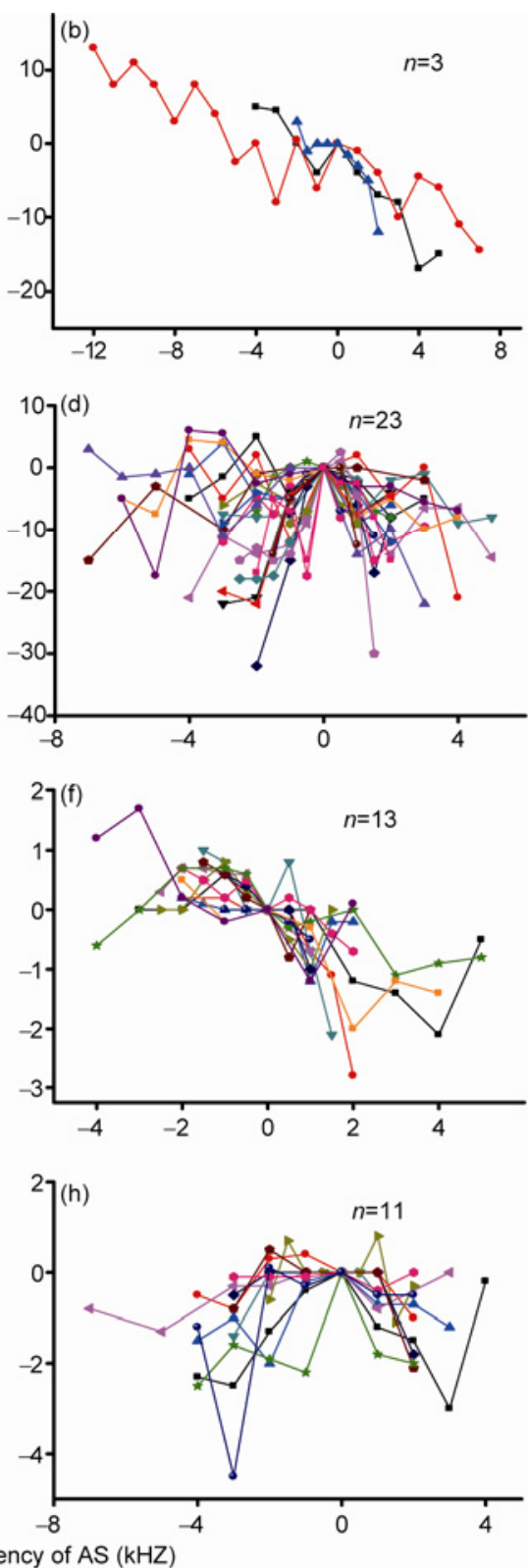

Figure 7 Four categories of $\Delta A$ and $\Delta L$ as a function of normalized frequencies $(n=37)$. The $\Delta A$ and $\Delta L$ increased monotonically with increasing frequency (type I; (a) and (e)), decreasing monotonically with increasing frequency (type II; (b) and (f)), changed non-monotonically with frequency in a "V" shape (type III; (c) and (g)) or changed non-monotonically in an inverse "V" shape (type IV; (d) and (h)). 
(e)) and increased with decreasing frequency for type II (Figure 7(b) and (f)). For type III, $\Delta A$ or $\Delta L$ changed non-monotonically with an increase in frequency, forming a "V" shape (Figure 7(c) and (g)), and type IV showed an inverse "V" shape (Figure 7(d) and (h)). For $\Delta A, 86.5 \%$ of neurons showed non-monotonic change (Figure $7(\mathrm{c})$ and (d)), with $62.2 \%$ of neurons showing an inverse "V" shape (type IV; Figure 7(d)). The remaining $13.5 \%$ of neurons showed monotonic change (Figure 7(a) and (b)). For $\Delta L$, $43.2 \%$ of neurons showed non-monotonic change (Figure $7(\mathrm{~g})$ and $(\mathrm{h}))$, while $56.8 \%$ neurons showed monotonic change (Figure 7(e) and (f)). Although these four categories of $\Delta A$ and $\Delta L$ changes could be identified, the change of $\Delta A$ and $\Delta L$ with the AS frequency varied between neurons.

Each parameter, $\Delta A$ or $\Delta L$, could not fully describe neuronal properties in terms of wire transmission and joint transduction. Therefore, we categorized neuronal properties using changes with AS frequency for both $\Delta A$ and $\Delta L$. Eleven types were identified in total (Figure 8(a)). In 23 out of 37 neurons, $\Delta A$ first increased then decreased. $\Delta L$ monotonically decreased with increasing AS frequency in $29.7 \%$ of neurons, non-monotonically increased then decreased in $18.9 \%$, and monotonically increased in $10.8 \%$. Of 9 neurons in which $\Delta A$ first decreased then increased, $\Delta L$ monotonically increased in $8.1 \%$, first increased then decreased in $8.1 \%$, and first decreased then increased in $8.1 \%$. All other types occurred with an incidence of $<5 \%$. The above categories may be related to some functions of sound processing. Then, the functional structures of wire transmission and joint transduction onto most sampled neurons, implied by the changes of $\Delta L$ and $\Delta A$ referenced to the $\mathrm{CF}$ response, were exhibited (Figure 8(b)). The most common type of $\Delta L$ and $\triangle A$ changes may be explained by the functional structure shown in Figure 8(b5). For this type, the $\Delta L$ changes monotonically while the $\Delta A$ changes non-monotonically as an inverse $\mathrm{V}$-shape. This means that transmission efficacy increased as the frequency of the AS increased, while the transduction efficacy increased when the frequency of the AS shifted away from the CF.

\section{Discussion}

In the present study, we investigated the ICC neuronal responses to acoustic pure tones of varying frequencies and amplitudes, using the $L-A$ curves obtained for different AS frequencies. There were three main findings. (1) The first spike latency is much more accurate, stable, and reliable than SC, and is a function of the AS amplitude. (2) The $L-A$ functions for different AS frequencies can be superimposed irrespective of their positions, confirming our hypothesis. (3) The AS-independent asymptotes $L_{0}$ and $A_{0}$ obtained from the fit of the $L-A$ plot, as well as the differences in the asymptotes $(\Delta L, \triangle A)$ between the $\mathrm{CF}$ and non-CF $L-A$ functions, may be suitable parameters to describe the efficacy of wire transmission and joint transduction of an ICC neuron.

The first finding supports previous results [11,14-17]. The latter two findings may provide a method to analyze the relative functional transduction and transmission by using the response at the $\mathrm{CF}$ as a reference.

\subsection{The implications of superimposition of $L-A$ functions}

The latency of the first acoustically evoked spike is determined by the integration of the sound envelope, which contains both amplitude and time course $[11,25,26]$. Both excitation and inhibition occur in the central auditory nervous system during the processing of acoustic information. However, we are only concerned with the acoustic information that is able to reach the recorded ICC neuron, that is, the excitatory integrated neuronal activities passed from one relay station to another in the ascending auditory pathway, to evoke acoustic perception. Since the excitatory integrated neuronal activities can be recorded extracellularly, the $L$ obtained by extracellular recording can be a used as a parameter that reflects the response properties of the auditory neuron.

Although the isofrequency $L-A$ functions of a neuron
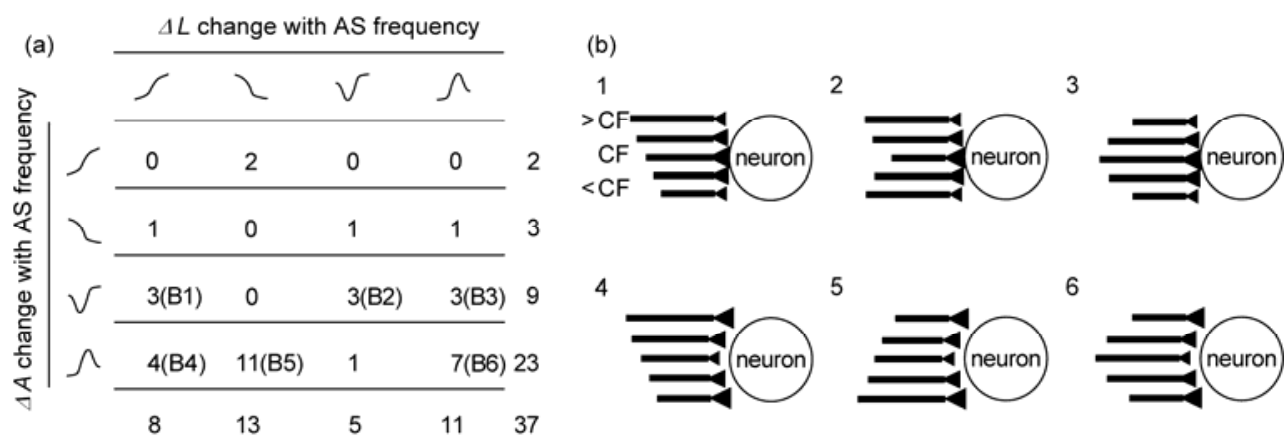

Figure 8 Summary of ICC neurons ( $n=37$ ) categorized by $\Delta A$ and $\Delta L$. (a) The number of neurons in each category (see text for details). (b) The functional wire and joint structures onto neurons implied by the changes of $\Delta L$ and $\Delta A$ relatively to the $\mathrm{CF}$ response. The length of the wire represents the transmission efficacy and the size of the joint represents the transduction efficacy. The central wire and joint represent the pathway activated by the AS at the CF of the recorded neuron. The upper two sets represent the pathways activated by the AS at $>\mathrm{CF}$, and the bottom two set represent those activated by the AS at $<\mathrm{CF}$. 
were different from each other at different AS frequencies (Figures 3(d), 4(b) and 4(d)), all $L-A$ functions were similar in shape and were superimposed when their $x-y$ position was adjusted (Figure 5(a)-(c)). This behavior of auditory neurons implies that sound amplitude processing obeys the same law (the curvature of the $L-A$ function as described by $\lambda$ and $\tau$ ) irrespective of AS frequency. Different AS frequencies can be processed referenced to one frequency, and this can be treated as filtering. Thus, the frequency processing of an ICC neuron might work as a filter. However, previous studies have also demonstrated that the $L-A$ functions in different auditory neurons can be superimposed by normalization of their positions to that of a reference neuron, indicating that the filtering is not achieved by the auditory neuron itself $[11,25,26]$. It is well known that the basilar membrane works as a filter. Thus, the behavior of ICC auditory neurons faithfully represents the physical laws of sound being transferred into bioelectric signals, and this may originate in the basilar membrane.

Regardless of the asymptotes $L_{0}$ and $A_{0}$ that determine the position of the $L-A$ curves, the superimposition of the $L-A$ functions suggests that auditory neurons are not concerned with the frequency input quality or the frequency input channel when processing the acoustic information. Only the input magnitude must be processed in the recorded neuron. The curvature of the superimposed $L-A$ functions, determined by $\lambda$ and $\tau$ (see Appendix I; $\lambda=b P_{0}^{-a}$ and $\tau=$ $20 / a \ln 10$, developed from Pieron's law [23]), might reflect the sensitivity of the recorded neuron, which may be determined by the properties of the neuronal membrane. This possibility should be tested by further experiments, such as whole-cell recording in vivo and in vitro.

All ICC neurons sampled in this study showed single-peak V-shape receptive fields. Therefore, we only tested the neuronal responses at frequencies ranging from $\mathrm{CF}-5$ to $\mathrm{CF}+5 \mathrm{kHz}$, which is limited to the central range of the receptive fields. The AS at other frequencies either evoked fewer neuronal responses requiring much higher sound levels, or induced no neuronal response at all. This might slightly decrease the success of data fitting. However, based on the consistent results obtained from ICC neurons with a large range of CFs $(6-40 \mathrm{kHz})$, the superimposition of $L-A$ functions for CF and non-CF stimuli is likely to be valid for a much larger range of frequencies within the neuron's receptive field.

\subsection{The meanings of $\Delta A$ and $\Delta L$}

As discussed above, the superimposition of the $L-A$ functions could be seen regardless of neurons $[11,25,26]$ and sound frequencies (Figure 5(a)-(c)). The important feature of the $L-A$ function is described by its curvature, i.e., the constants $\lambda$ and $\tau$. Thus, $\lambda$ and $\tau$ are determined by the response properties of the basilar membrane, which works as a filter. In contrast, the position of the $L-A$ function is de- termined by the horizontal and vertical asymptotes, that is, the constant $L_{0}$ and $A_{0}$, or the differences relative to a reference, expressed by $\Delta A$ in $\mathrm{dB}$ SPL and $\Delta L$ in ms. As discussed in the Introduction, $L_{0}$ and $A_{0}$ may represent the efficacy of wire transmission and joint transduction, respectively. Therefore, $\Delta L$ and $\Delta A$ for a given non-CF channel reflect the differences in transmission and transduction efficacy compared to the $\mathrm{CF}$ channel.

When $\Delta L$ is zero, the non-CF channel carries the acoustic information with a similar transmission efficacy to the $\mathrm{CF}$ channel, but they do not necessarily represent the same channel. However, the channels for $\mathrm{CF}$ and non-CF acoustic information processing are likely to be different when $\Delta L \neq$ 0 or $\Delta A \neq 0$. When $\Delta A$ and $\Delta L$ of a neuron are plotted against AS frequency, the functions (Figures 5(d)-(f) and 7) may represent a neuron's relative functional structure of the wire and joint. The wire transmission reflected by $\Delta L$ includes transmission of the acoustic signal in the air, the cochlear liquid, the synaptic gap, and the axon [17]. The latter two can vary with different frequencies, whereas the first two are relative independent of sound frequencies. In contrast to $\Delta L, \Delta A$ represents the sensitivity of the joint transduction, which includes the summation of pre-synaptic neurotransmitter release and postsynaptic membrane responses for all activated synapses. In summary, $\Delta L$ can be a functional measurement of the difference in the length of the wire, i.e., the sum of the involved synapses, compared to a reference such as the CF channel (Figure 8(b), the lines), while $\Delta A$ can be a functional measurement of the strength of the joint, i.e., the sum of the sensitivity of the involved synapses (Figure 8(b), the dark triangles).

\subsection{The implications of the functional structure of in- puts to ICC neurons as estimated by $\Delta L$ and $\Delta A$}

By taking $\Delta L$ and $\triangle A$ as the measurements of the functional structure of a frequency channel, we plotted $\Delta A$ and $\Delta L$ of all studied ICC neurons against the AS frequency. The results showed the $\Delta L$ and $\Delta A$ were different for different frequency channels and neurons (Figures 7 and 8(a)). This suggests that differences in neuronal responses are always caused by activation of different pathways, and that the auditory pathways that process different sound frequencies are diverse. The functional structure of the wire and joint of a simplified frequency channel could be categorized into 11 types (Figure 8(a)). Six of these were relatively frequently observed in the studied neurons. The functional structures of these neurons were schematically demonstrated in Figure 8 (b). Because $\triangle A$ indicates the strength of the summated synapses, and $\Delta L$ indicates the total length of the fibers (including synaptic gaps), the arrangement of the frequency channels to a neuron is clearly outlined. We can easily observe the regularities of the neuron's functional structure. However, the exact function of these neurons in sound information processing should be tested in further studies. 
This work was supported by the National Natural Science Foundation of China (31171059, 30930039 and 30970982) and Program for Changjiang Scholars and Innovative Research Team in University (IRT1142).

1 Heil P. First-spike latency of auditory neurons revisited. Curr Opin Neurobiol, 2004, 14: 461-467

2 Nieuwenhuys R. Anatomy of the auditory pathways, with emphasis on the brain stem. Adv Otorhinolaryngol, 1984, 34: 25-38

3 Demanez J P, Demanez L. Anatomophysiology of the central auditory nervous system: Basic concepts. Acta Otorhinolaryngol Belg, 2003, 57: 227-236

4 Malmierca M S. The structure and physiology of the rat auditory system: An overview. Int Rev Neurobiol, 2003, 56: 147-211

5 Cant N B, Benson C G. Parallel auditory pathways: Projection patterns of the different neuronal populations in the dorsal and ventral cochlear nuclei. Brain Res Bull, 2003, 60: 457-474

6 VanRullen R, Guyonneau R, Thorpe S J. Spike times make sense. Trends Neurosci, 2005, 28: 1-4

7 Chase S M, Young E D. First-spike latency information in single neurons increases when referenced to population onset. Proc Natl Acad Sci USA, 2007, 104: 5175-5180

8 Johansson R S, Birznieks I. First spikes in ensembles of human tactile afferents code complex spatial fingertip events. Nat Neurosci, 2004, 7: 170-177

9 Perez-Orive J, Mazor O, Turner G C, et al. Oscillations and sparsening of odor representations in the mushroom body. Science, 2002, 297: 359-365

10 de Charms R C, Merzenich M M. Primary cortical representation of sounds by the coordination of action-potential timing. Nature, 1996, 381: 610-613

11 Heil P. Auditory cortical onset responses revisited. I. First-spike timing. J Neurophysiol, 1997, 77: 2616-2641

12 Meister M, Berry M J. The neural code of the retina. Neuron, 1999, 22: 435-450

13 Mehta M R, Lee A K, Wilson M A. Role of experience and oscillations in transforming a rate code into a temporal code. Nature, 2002, 417: 741-746

14 Mickey B J, Middlebrooks J C. Representation of auditory space by cortical neurons in awake cats. J Neurosci, 2003, 23: 8649-8663

15 Brugge J F, Anderson D J, Hind J E, et al. Time structure of discharges in single auditory nerve fibers of the squirrel monkey in response to complex periodic sounds. J Neurophysiol, 1969, 32: 386401

16 Tan X, Wang X, Yang W, et al. First spike latency and spike count as functions of tone amplitude and frequency in the inferior colliculus of mice. Hear Res, 2008, 235: 90-104

17 Liang F, Yang W, Zheng X, et al. Response property of inferior collicular neurons inherited from peripheral origin in mouse. Brain
Res, 2011, 1369: 46-59

$18 \mathrm{Lu} \mathrm{T}$, Wang X. Information content of auditory cortical responses to time-varying acoustic stimuli. J Neurophysiol, 2004, 91: 301-313

19 Rhode W S, Smith P H. Encoding timing and intensity in the ventral cochlear nucleus of the cat. J Neurophysiol, 1986, 56: 261-286

20 Qiu Q, Tang J, Yu Z, et al. Latency represents sound frequency in mouse IC. Sci China Ser C-Life Sci, 2007, 50: 258-264

21 Aitkin L M, Anderson D J, Brugge J F. Tonotopic organization and discharge characteristics of single neurons in nuclei of the lateral lemniscus of the cat. J Neurophysiol, 1970, 33: 421-440

22 Aitkin L M, Dunlop C W. Interplay of excitation and inhibition in the cat medial geniculate body. J Neurophysiol, 1968, 31: 44-61

23 Pieron H. Nouvelles recherches sur l'analyse du temps de latence sensorielle en function des intenste's excitatrices. Ann Psychol, 1920, 22: $58-142$

24 Fishbach A, Nelken I, Yeshurun Y. Auditory edge detection: A neural model for physiological and psychoacoustical responses to amplitude transients. J Neurophysiol, 2001, 85: 2303-2323

25 Heil P, Neubauer H. Temporal integration of sound pressure determines thresholds of auditory-nerve fibers. J Neurosci, 2001, 21: 7404-7415

26 Heil P, Neubauer H. A unifying basis of auditory thresholds based on temporal summation. Proc Natl Acad Sci USA, 2003, 100: 61516156

27 Krishna B S. A unified mechanism for spontaneous-rate and firstspike timing in the auditory nerve. J Comput Neurosci, 2002, 13: 71-91

28 Meddis R. Auditory-nerve first-spike latency and auditory absolute threshold: A computer model. J Acoust Soc Am, 2006, 119: 406-417

29 Casseday J H, Fremouw T, Covey E. The inferior Colliculus: A hub for the central auditory system. In: Oertel D, Fay R R, Popper A N, eds. Integrative Functions in the Mamalian Auditory Pathway. Berlin Heidelberg \& New York: Springer, 2002. 238-318

30 Tang J, Xiao Z J, Shen J X. Delayed inhibition creates amplitude tuning of mouse inferior collicular neurons. Neuroreport, 2008, 19: 1445-1449

31 Keithley E M, Feldman M L. The spiral ganglion and hair cells of Bronx waltzer mice. Hear Res, 1983, 12: 381-391

32 Sivaramakrishnan S, Sterbing-D'Angelo S J, Filipovic B, et al. GABA(A) synapses shape neuronal responses to sound intensity in the inferior colliculus. J Neurosci, 2004, 24: 5031-5043

33 Sutter M L, Schreiner C E. Topography of intensity tuning in cat primary auditory cortex: Single-neuron versus multiple-neuron recordings. J Neurophysiol, 1995, 73: 190-204

34 Stafford T, Gurney K N. The role of response mechanisms in determining reaction time performance: Pieron's law revisited. Psychon Bull Rev, 2004, 11: 975-987

35 Aitkin L, Martin R. Neurons in the inferior colliculus of cats sensitive to sound-source elevation. Hear Res, 1990, 50: 97-105

Open Access This article is distributed under the terms of the Creative Commons Attribution License which permits any use, distribution, and reproduction in any medium, provided the original author(s) and source are credited.

\section{Appendix I}

Pieron's empirical law [23]:

$$
R T-R T_{\min }=b P^{-a} \text {, }
$$

where $R T$ and $R T_{\min }$ represent the response time and minimum response time, respectively (they are expressed as $L$ and $L_{0}$ in the present study). $a$ and $b$ are constants, while $P$ is the sound pressure in Pascal. The sound pressure is converted into sound amplitude in $\mathrm{dB}$ SPL as follows:

$$
\begin{aligned}
& A=20 \log \left(P / P_{\min }\right), \\
& A_{0}=20 \log \left(P_{0} / P_{\min }\right), \\
& A-A_{0}=20 \log \left(P / P_{0}\right),
\end{aligned}
$$


where $A$ is the amplitude of an acoustic stimulus in $\mathrm{dB}$ SPL, $P_{0}$ is the minimum sound pressure required to activate the recorded ICC neuron, while the $P_{\min }$ is the minimum sound pressure. Then,

$$
P=P_{0} e^{\left(A-A_{0}\right) \ln 10 / 20} .
$$

When $P$ in Pieron's law is replaced, the function changes into

$$
\begin{aligned}
& L-L_{0}=b\left(P_{0} e^{\left(A-A_{0}\right) \ln 10 / 20}\right)^{-a}, \\
& L-L_{0}=b P_{0}^{-a} e^{-\left(A-A_{0}\right) / 20 /(a \ln 10)} .
\end{aligned}
$$

If $\lambda=b P_{0}^{-a}$ and $\tau=20 / a \ln 10$, then Equation (2) used in this study can be developed from Pieron's law:

$$
L-L_{0}=\lambda e^{-\left(A-A_{0}\right) / \tau} \text {. }
$$

